FRI0729-HPR RHEUMATOLOGY ADVANCED NURSE PRACTITIONERS TREAT TO TARGET PERSON CENTERED CARE: IRELAND'S POLICY FRAMEWORK.

P. Minnock ${ }^{1}$, A.-M. Ryan ${ }^{2}$ on behalf of Irish Rheumatology Nursing Forum. ${ }^{1}$ Rheumatic Musculoskeletal Disease Unit, Our Lady's Hospice and Care Services; ${ }^{2}$ Deputy Chief Nursing Officer, Department of Health, Dublin, Ireland

Background: Advanced practice refers to a registered nurse, educated to master's degree level, with the expert knowledge base, complex decision-making skills and clinical competencies for expanded practice beyond that of the first leve nurse. Advanced practice characteristics are shaped by the speciality, local context and/or country of practice. Countries, as well as specialty areas are at different stages in the development of legislation, scope of practice, roles, responsibilities, education and clinical preparation. Rheumatology nursing development continues apace internationally.

Objectives: To bring rheumatology advanced nurse practitioners (ANPs) to the forefront in the delivery of quality person-centered care, working to enhance patient outcomes and reduce the personal and societal burden of rheumatic musculoskeletal diseases.

Methods: The Irish Rheumatology Nursing Forum proposed a business case for the development of advanced nurse practitioner posts to implement, as a standardised approach to care nationally, the therapeutic strategy of treat to target for patients with inflammatory arthritis. This was endorsed by the Irish Society for Rheumatology and approved by the Rheumatology National Clinical Programme in 2015. Subsequently this proposal was chosen by the Chief Nursing Office as a demonstrator project for the Department of Health $(\mathrm{DOH})$ draft policy to raise the critical mass of ANPs in healthcare delivery.

Results: In late 2017, the DOH allocated 22 new ANP posts to rheumatology services countrywide, aligned to the national integrated care programme for the prevention and management of chronic disease. These 22 candidate ANPs are now completing advanced practice education at MSc level run by a consortium of Irish universities (University College Dublin, Cork, Galway, and Trinity College Dublin). Supervision of the requisite 500 clinical hours at advanced practice level is being provided by local consultant rheumatologist in partnership with the universities. Local teams of key service, nursing, medical, and academic personnel have been established across all centres to oversee project development; implementation; utilisation of robust evaluation criteria to capture clinical impact and cost-effectiveness. Initial evaluation will focus on key performance indicator's related to patient access such as i) ratio of new patients seen to ANPs, ii) new: return attendance ratio, iii) percentage of referrals seen within three months, iv) percentage of non-attended appointments. Intermediate-long-term evaluation will encompasses patient care and health care outcome through evaluation of all nursing interventions such as health assessments; medication prescribing and optimisation; patient education; health promotion; comorbidity screening; referral to other professionals; ordering of investigations; patient and staff satisfaction survey data. Quality of care will be evaluated guided by published quality care indicators. Patient outcome will be evaluated using appropriate nursing sensitive and validated disease activity scores and patient reported outcome measures. Realtime data collection using a specifically commissioned epr will ensure cANPs are supported by the appropriate technology to treat to target.

Conclusions: ANP-led care underpinned by evidence based practice and guidelines, continues to grow as a model of care delivery in rheumatology.

Disclosure of Interest: None declared

DOI: 10.1136/annrheumdis-2018-eular.4010

\section{FRI0730-HPR THE CONCEPT OF PATIENT CENTERED CARE IN SPONDYLOARTHRITIS BASED ON A MULTIDISCIPLINARY MODEL}

P. Santos-Moreno ${ }^{1}$, L. Villarreal ${ }^{2}$, D. Buitrago-Garcia ${ }^{3}$, M. Cabrera ${ }^{2}$.

${ }^{1}$ Rheumatology; ${ }^{2}$ Health Services; ${ }^{3}$ Epidemiology, Biomab, Center For Rheumatoid Arthritis, Bogota, Bogota, Colombia

Background: Spondyloarthritis (SpA) is one the most prevalent musculoskeletal disease in the Americas, with an estimated prevalence of $0.5 \%$. This group of patients presents a number of unmet needs for accessibility to the consultation, diagnosis and adequate treatments. That for this reason it is necessary to develop a program of Centers of Excellence (CoE), which allows answer to these needs and at the same time to add values for our health systems.

Objectives: The aim was to create a program with pilot SpA centers initially in some countries that operate under the scheme of $\mathrm{CoE}$, as they are already delineated in projects like REAL-PANLAR for rheumatoid arthritis. In a second phase and under the auspices of PANLAR (Panamerican league of associations for rheumatology) create a Pan American Network of Centers of Excellence in SpA (CESPA).

Methods: We performed a systematic review of the literature in global and regional databases (Pubmed, Medline, Scopus, Lilacs), in order to search information on this research question-hypothesis. Subsequently and under a Delphi-modified methodology and consensus of involved rheumatologists lay the conceptual bases on this particular subject - the Centers of Excellence in SpA (CESPA). As a result of the above was defined as should be a CESPA.

Results: In accordance with the principles of creation and operation of the $\mathrm{CoE}$ in particular, specific themes were developed by a coordinator who, after a review of the literature, presented a specific proposal on every particular topic that was discussed and then voted on and implemented swithin the CESPA concepts. A CESPA must have: 1 . Screening Clinic and Early Diagnosis of SpA: the purpose is to rule out false positives of disease and to diagnose early SpA; 2 . Model of patient-centered care: a model of frequencies of care should be implemented for $\mathrm{SpA}$ patients from the perspective of the different specialties involved in the multidisciplinary team; 3. Laboratories and images: conventional laboratory tests as having a minimum of 3-4 times a year and each patient will have conventional $X$ ray images of cervical and lumbosacral column at the beginning and then once a year. Ultrasound of entheses: upon admission to the program and then with a certain periodicity (annual) or depending of sensitivity to change (OMERACT). The same applies to the subject of MRI of sacroiliac joints and column for both diagnosis and follow-up; 4. Clinical guideline on SpA should be adapted and customized to the particular realities of some of our countries; 5 . There must be a management (disease management) program that must include Structure, Process and Result Indicators to define CESPA as Standard, Optimal and Model; 6. There must be standardized education and research programs for patients and physicians at the center

Conclusions: There is a global need to develop CESPAs, in order to define treatment targets type T2T-SpA, which would improve clinical outcomes and avoid so much disability and health economic costs.

\section{REFERENCES :}

1. Santos-Moreno $P$, et al. REAL-PANLAR Project for the Implementation and Accreditation of Centers of Excellence in RA Throughout Latin America. J Clin Rheumatol 2015 Jun;21(4):175-80.

2. Santos-Moreno $\mathrm{P}$, et al. From the model of integral attention to the creation of centers of excellence in RA. Clin Rheumatol. 2015 Mar;34(Suppl 1): S71-7.

Disclosure of Interest: None declared DOI: 10.1136/annrheumdis-2018-eular.7469

FRIDAY, 15 JUNE 2018

HPR Professional education, training and competencies

\section{FRI0731-HPR SWITCH MANAGEMENT BETWEEN SIMILAR BIOLOGICAL MEDICINES, A COMMUNICATION INFORMATION GUIDE FOR NURSES}

J.E. Voorneveld-Nieuwenhuis ${ }^{1}$, L. Moortgat ${ }^{2}$, M. Pavic Nikolic ${ }^{3}$, P. Crombez ${ }^{4}$ B. Oomen ${ }^{5}$ on behalf of European Specialist Nurses Organisations (ESNO): representatives of five organisations: Oncology, Diabetes, Dermatology, Rheumatology and Inflammatory Bowel Diseases are involved.. ${ }^{1}$ Rheumatology, Maasstad Hospital Rotterdam, Rotterdam, Netherlands; ${ }^{2}$ Gastroenterology, AZ Delta, Roeselare, Belgium; ${ }^{3}$ Rheumatology, University Medical Centre, Ljubljana, Slovenia; ${ }^{4}$ Hematology, Jules Bordet Institute; ${ }^{5}$ ESNO, Brussels, Belgium

Background: Biologics are used in the field of rheumatology but are also important in other fields such as oncology and gastroenterology. Over the last few years, biosimilar versions of many biologicals have been launched. Patients may be switched depending on local rules and appointments. To provide support and information for nurses working with patients who are switching between similar biological medicines ESNO has taken the initiative to develop an information guide for nurses.

Nurses can take the lead in implementing the transition between branded and biosimilar biologic medicines. This includes managing the process before, during and after the switch.

Objectives: The guide was developed to provide support and information for nurses working with patients who are switching between similar biological medicines

The guide provides examples of projects and best practices based on different specialties to increase trust in biological medicines including biosimilars. Its aim is to contribute to the safe use of and trust in biologic medicines, and give nurses the tools to implement switching decisions in a clinical context and deal with patient concerns, drawing on the learnings from real-life experiences.

Methods: We collected previous experiences from nurses with switching biologics. We also developed a roadmap for how to inform patients, a set of frequently asked questions (FAQ) and points to consider when switching. 
Results: The guide is written in English and will be translated into the 23 different languages of the EU.

With information about:

- The definitions of biological and biosimilar medicines.

- Switching and substitution.

- The benefits of biosimilars

- The nurses role in managing the exchange between similar biological medicine.

The document also includes flow charts for switch implementation, follow up and support.

Conclusions: Switching between similar biologicals opened new chapter in which nurses play a crucial role in communicating with patients and providing support and reassurance, before, during and particularly after the switch. This is build on nurses' many years of education, and their experience with patients in different situations. It is a process that requires time, patience and care.

Patients may be concerned about changes in biologic medicines, and will have a lot of questions. Positive language is important in answering these questions, to provide confidence and reassurance. Patients need to know that their healthcare professionals understand the reasoning behind the change and are confident that it is the right thing to do. To avoid confusion, the team of nurses and other healthcare professionals should have a consistent explanation that is used by all.

\section{REFERENCES:}

1. European Commission. What I need to know about biosimilar medicines: Information for patients 2016.

2. European Medicines Agency and European Commission. Biosimilars in the EU: Information guide for healthcare professionals 2017.

3. Read C. Specialist nurses support cost effective drugs for treatment. HSJ 2017.

Disclosure of Interest: J. Voorneveld-Nieuwenhuis: None declared, L. Moortgat: None declared, M. Pavic Nikolic: None declared, P. Crombez: None declared, B. Oomen Grant/research support from: This document has been created with funding from Medicines for Europe and EFPIA

DOI: 10.1136/annrheumdis-2018-eular.2376

\section{FRI0732-HPR RHEUMATOLOGY NURSE SPECIALISTS AND CORTICOSTEROID PRESCRIBING - DOES IT CONFORM TO EULAR GUIDELINES}

J. Begum ${ }^{1}$, M.K. Nisar ${ }^{1} .{ }^{1}$ Rheumatology, Luton \& Dunstable University Hospital, Luton, United Kingdom

Background: The rheumatology nurse specialist (RNS) plays a central role in the multi-professional rheumatology team. Delivering corticosteroid (CS) therapy to patients is an area where there is little understanding of RNSs confidence in managing them.

Objectives: Considering CSs are a cornerstone of treating rheumatic diseases where RNSs are invariably involved, we undertook a pilot survey to understand the present climate of RNSs prescribing of corticosteroids in their practice.

Methods: A focus group discussion was held at South West regional meeting to ascertain the minimum level of understanding required to successfully deliver CS therapy to patients. It was centred on EULAR recommendations. Nine items were identified based on three main themes - safe prescribing, optimal dosing and prevention of complications. A questionnaire was created based on this discussion and all participants of the meeting were surveyed.

Results: There are 21 centres providing rheumatology services in the South West England. All were represented in 30 participants of the survey. Median age of the nursing establishment was 48 years (mean 47 year, range 27-60 years). Only $6 / 30(20 \%)$ were nurse prescribers.

$14(47 \%)$ did not feel comfortable advising patients on adjusting their CS dose. Only four (13\%) had any patient group directive in place at their trust to enable them to amend CS dose for non-medical prescribers. $11(36 \%)$ considered CS to be disease modifying therapy in inflammatory arthritis. 17 (56\%) employed CS therapy as part of early arthritis management protocol. 4/30 (13\%) considered prednisolone equivalent dose of $\geq 10 \mathrm{mg} /$ day safe in long term and seven $(23 \%)$ would be happy to utilise $120 \mathrm{mg}$ IM depomedrone monthly as necessary. 10 $(33 \%)$ were unaware of therapeutic co-intervention for CS related osteoporosis risk and $21(70 \%)$ were not employing any fracture risk stratification tools.

Conclusions: This pioneering initiative highlights a wide variation in the prescription standards of a key job provision. Very few units have independent nurse prescribers. Others lack patient group directive to at least enable non-medical prescribers i.e. RNSs to safely amend CS therapy prescribed by a rheumatologist. Less than a quarter of those surveyed actually consider CSs to have any diseasemodifying role. Rather worryingly, some do not even recognise the safe long-term CS dose and willing to offer high doses periodically. Though most know the concomitant therapeutic options to mitigate against osteoporosis, few are actually assessing fracture risk thereby unlikely to offer the appropriate interventions. In conclusion, there is wide variation in the service provision of RNSs. This can potentially have a negative impact on effort to promote safer use of CSs in the management of inflammatory rheumatic diseases. There is a need for improving training standards to help deliver good quality rheumatology professionals of the future and ensure safe and effective drug interventions.

Disclosure of Interest: None declared

DOI: 10.1136/annrheumdis-2018-eular.1299

\section{FRI0733-HPR AN INTERACTIVE COURSE ON EXERCISE THERAPY FOR KNEE OSTEOARTHRITIS AND COMORBIDITY: A FEASIBILITY STUDY}

M. De Rooij ${ }^{1}$, M. van der Leeden ${ }^{2}$, M. van der Esch ${ }^{1}$, W.F. Lems ${ }^{3}$, J. Meesters ${ }^{4}$, W. F. Peter ${ }^{1}$, L.D. Roorda ${ }^{1}$, M. Terbraak ${ }^{5}$, T. Vredeveld ${ }^{5}$, T.P. Vliet Vlieland ${ }^{4}$, J. Dekker ${ }^{2} .{ }^{1}$ Department of Rehabilitation, Reade, center of rehabilitation and rheumatology; ${ }^{2}$ Department of Rehabilitation; ${ }^{3}$ Department of Rheumatology, VU Medical Centre, Amsterdam; ${ }^{4}$ Department of Orthopedics, Leiden University Medical Center (LUMC), Leiden; ${ }^{5}$ Faculty of Health, ACHIEVE Centre of Expertise, Amsterdam University of Applied Sciences, Amsterdam, Netherlands

Background: A structured, tailored exercise therapy strategy was found to significantly improve physical functioning, reduce pain and was safe for patients with knee OA and severe comorbidity. The intervention was performed in a specialized, secondary care center. Before the intervention can be implemented in primary care, appropriate education of physiotherapists (PTs) as well as insight into barriers and facilitators for using the protocol in primary care is needed.

Objectives: This study aimed to 1) evaluate the effect of an interactive course on the exercise therapy strategy for patients with OA and comorbidity for PTs working in primary care; and 2) map facilitators and barriers for applying the protocol in primary care.

Methods: A pre-posttest study was performed among PT's who were working in primary care. PTs were offered a postgraduate blended educational course consisting of an e-learning lecture (7 hours study load) and two interactive workshops (each 3 hours study load). Measures of effectiveness included a questionnaire on knowledge (60 multiple choice questions) before (T0) and two weeks after the course (T1) and a patient vignette to measure clinical reasoning (nine open ques tions) before the course (T0) and six months after the course (T2). Facilitators and barriers for using the protocol were measured at T2 by means of a 27 item questionnaire (each item was scored on a 5-point Likert scale, ranging from 0 totally agree to 4 totally disagree).

Results: Thirty-four PTs were included. Fourteen out of 34 PTs had treated at least one patient with knee OA and comorbidity according the protocol. Statistically significant improvements were found, both for knowledge levels between baseline and $\mathrm{T} 1(\mathrm{~N}=34)(\mathrm{p}<0.00)$, and for clinical reasoning between baseline and T2 $(N=34)(p<0.00)$. With regard to facilitators to implement the protocol, the majority of PTs found the protocol feasible in daily practice $(68 \%)$ and to be supportive regarding clinical reasoning and clinical decision making $(77 \%)$. Perceived barriers for implementation included the small number of patients with $O A$ and severe comorbidity being referred or referring themselves. Of the therapist who actually treated patients according to the protocol, $86 \%$ indicated that the protocol was applicable in their daily clinical practice and that they perceived to have sufficient knowledge $(71 \%)$ and skills $(64 \%)$ to apply the protocol. Other barriers indicated by PTs were the limited number of treatment reimbursement by the insurance companies $(65 \%)$ and a suboptimal collaboration with general practitioners and physicians (65\%).

Conclusions: An interactive educational course on exercise therapy for knee OA patients with comorbidity proved to be effective in improving knowledge and clinical reasoning skills of primary care PTs. Main barriers for protocol use included limited referrals of patients with knee OA and severe comorbidity to PTs and limited number of treatment reimbursement by insurance companies. For larger scale implementation these barriers should be solved.

Disclosure of Interest: None declared

DOI: 10.1136/annrheumdis-2018-eular.5144 